\title{
Factores asociados a la satisfacción laboral del profesorado universitario
}

Factors associated to the job satisfaction of university teaching staff

\author{
Volumen 21, Número 1 \\ Enero - Abril \\ pp. 1-24
}

Cesar H. Limaymanta

Osbaldo Turpo-Gebera

Citar este documento según modelo APA

Limaymanta, Cesar H. y Turpo-Gebera, Osbaldo. (2021). Factores asociados a la satisfacción laboral del profesorado universitario. Revista Actualidades Investigativas en Educación, 21(1), 1-24. Doi. 10.15517/aie.v21i1.42494 


\title{
Factores asociados a la satisfacción laboral del profesorado universitario \\ Factors associated to the job satisfaction of university teaching staff
}

\author{
Cesar H. Limaymanta ${ }^{1}$ \\ Osbaldo Turpo-Gebera²
}

\begin{abstract}
Resumen: El ámbito laboral debe estar ligado a lo que nos motiva y nos apasiona, dado que involucra gran parte de nuestras vidas. El propósito de este artículo se centró en analizar los factores asociados a la satisfacción laboral del profesorado de una universidad pública y de otra privada ubicadas en Lima, Perú. Se empleó la metodología cuantitativa con diseño no experimental de corte transversal y nivel de estudio correlacional. Los datos fueron recolectados en 2018 a través de un cuestionario adaptado sobre satisfacción laboral, que cumple con las propiedades de validez y confiabilidad. La muestra estuvo conformada por 98 docentes en la categoría de ordinarios. El tratamiento de los datos se basó en el uso de las técnicas de la estadística descriptiva e inferencial, como la prueba $t$, U de Mann-Whitney, ANOVA y la correlación de Spearman. Los resultados evidencian que el reconocimiento, las condiciones de trabajo, el logro, la compensación y las oportunidades de promoción se asocian con la satisfacción laboral, mientras que los factores sociodemográficos y académicos, como grupo etario, sexo, formación académica y categoría laboral no se asocian con ella. Además, se evidenció que no existe diferencia entre los niveles de satisfacción laboral del personal docente de ambas universidades. Por último, se presentan posibles alcances y propuestas para mejorar los niveles de satisfacción laboral del profesorado universitario.
\end{abstract}

Palabras clave: satisfacción laboral, universidad, compensación, docentes

\begin{abstract}
The work environment must be linked to what motivates and excites us, since it involves a large part of our lives. The purpose of this article focused on analyzing the factors associated with the job satisfaction of the teaching staff of a public university and a private university located in Lima, Peru. The quantitative methodology was used with a non-experimental cross-sectional design and level of correlational study. The data were collected in 2018 through an adapted questionnaire on job satisfaction, which meets the properties of validity and reliability. The sample was made up of 98 professors in the category of ordinary. The treatment of the data was based on the use of the techniques of descriptive and inferential statistics, such as the t test, $U$ of Mann-Whitney, ANOVA and the correlation of Spearman. The results show that recognition, working conditions, achievement, compensation, and promotion opportunities are associated with job satisfaction, while sociodemographic and academic factors, such as age group, sex, academic background, and job category are not associated with it. In addition, it was shown that there is no difference between the levels of job satisfaction of the professors of the two universities. Finally, possible scopes and proposals to improve the levels of job satisfaction of university teaching staff are presented.
\end{abstract}

Keywords: job satisfaction, university, compensation, professors

\footnotetext{
${ }^{1}$ Docente en la Universidad Nacional Mayor de San Marcos, Lima, Perú. Doctorado en Ciencias de la Educación por la Pontifica Universidad Católica del Perú, Lima, Perú. Dirección electrónica: halley.limaymanta@pucp.edu.pe ORCID: https://orcid.org/0000-0002-8797-4275

2 Docente en la Pontificia Universidad Católica del Perú y en la Universidad Nacional de San Agustín, Lima, Perú. Doctorado en Educación por la Universidad Nacional Mayor de San Marcos, Lima, Perú. Dirección electrónica: oturpog@pucp.pe ORCID: https://orcid.org/0000-0003-2199$561 X$
}

Artículo recibido: 24 de junio, 2020

Enviado a corrección: 7 de julio, 2020

Aprobado: 19 de octubre, 2020 


\section{Introducción}

Desde la publicación de las monografías Management and the Worker, de Roethlisberger y Dikson, y Job Satisfaction, de Hoppock, en los años 30, la investigación sobre las actitudes laborales se ha incrementado notablemente (Locke, 1969). Desde entonces, los teóricos de las ciencias sociales y los investigadores en psicología, sociología organizacional, semiótica, antropología estructural, economía, comportamiento organizacional y gestión de recursos humanos han dedicado gran atención al constructo de la satisfacción laboral (SL, en adelante) (Cranny, Smith y Stone, 1992). Diversos estudios han establecido que el primer paso para mejorar la SL consiste en determinar sus causas y correlaciones (Cranny et al., 1992). Además, para Álvarez-Flores (2007), es necesaria la consideración de los factores que la motivan, es decir, aquellos determinantes o condicionantes del cambio. Entre ellos, los factores sociodemográficos, tales como estado civil, sexo y edad; y los factores laborales propiamente dichos, como el salario, las promociones, el reconocimiento, los beneficios, las condiciones de trabajo, la supervisión y la satisfacción con colegas de trabajo y la organización.

En cuanto a la SL en la profesión docente, Mapesela y Hay (2006) manifiestan que el constructo es emergente entre las instituciones educativas, y de gran consideración en las universidades (Rodríguez, Núñez y Cáceres, 2010). Está asociada a la búsqueda de la calidad y la acreditación de las carreras profesionales. En el ámbito peruano, el artículo 6 de la Ley Universitaria N. ${ }^{\circ} 30220$ (2014) estipula que las universidades peruanas forman, de manera integral, profesionales de alta calidad. En ese contexto, el profesorado actúa como eje central de la formación de las nuevas generaciones que se integrarán a la fuerza de trabajo (DuchePérez, Gutiérrez-Aguilar y Paredes-Quispe, 2019). Esto demuestra que la función docente y, por tanto, su SL son relevantes. Sin embargo, en el contexto peruano existen escasos estudios sobre los factores asociados a la SL del personal de enseñanza universitaria (Álvarez-Flores, 2007; Tinoco, Quispe y Beltrán, 2014), por lo que resulta necesario identificar los factores laborales y sociodemográficos pertinentes, además de comparar los niveles de la SL del cuerpo docente de dos o más universidades (El Badawy y Magdy, 2015; Khalid, Zohaib y Mahmood, 2012; Kenney, 2010). En respuesta a este panorama, el problema de investigación formulado es: ¿Cuáles son los factores que configuran la SL en el profesorado de una universidad pública y una universidad privada ubicadas en Lima Metropolitana?

Para responder al problema, se planteó el objetivo general: Explicar los principales factores que configuran la SL del profesorado de una universidad pública y de una universidad privada ubicadas en la ciudad de Lima Metropolitana. Este objetivo se alcanza a partir de los 
tres objetivos específicos: 1) identificar el nivel de SL del profesorado que labora en la Facultad de Derecho de una universidad pública y en la de una universidad privada ubicadas en Lima Metropolitana; 2) comparar el nivel de SL del profesorado que labora en la Facultad de Derecho de una universidad pública y en la de una universidad privada ubicadas en Lima Metropolitana; y 3) determinar los factores asociados (condiciones sociodemográficas y laborales) a la SL del profesorado que labora en la Facultad de Derecho de una universidad pública y en la de una universidad privada ubicadas en Lima Metropolitana. El logro de los objetivos permitirá generar propuestas orientadas al desarrollo de las instituciones superiores y al Estado peruano para mejorar el nivel de SL del personal docente universitario.

\section{Referentes teóricos}

La satisfacción o insatisfacción laboral ha sido abordada desde diversas perspectivas (Cantón-Mayo y Téllez-Martínez, 2016), sea como reacciones emocionales complejas hacia el trabajo (Locke, 1969) o como constructo constituido por criterios racionales (Frías, 2006). Ambos enfoques configuran el concepto de SL, puesto que los planos de actuación están muy relacionados y son ampliamente considerados en su definición.

La SL es un estado emocional y placentero que resulta de la valoración propia del trabajo (Park y Ramos, 2018; Toker, 2011). También se explica desde dos ópticas: i) la unidimensional, entendida como un concepto global; y ii) la multidimensional, al considerar diversos factores, componentes o dimensiones (Ssesanga y Garrett, 2005; Rodríguez et al., 2010). De manera integral, Guëll (2014) configura la SL a partir de tres componentes: cognitivo, afectivo y comportamental, manifestados a través de las percepciones, sentimientos y actitudes, respectivamente. De acuerdo con esta perspectiva, la SL del profesorado es un constructo relevante que resulta de la comparación entre lo que se posee y aquello que se debería tener, según un determinado horizonte de expectativas en el ambiente laboral educativo. La distancia de comparación define el nivel de satisfacción (Rodríguez et al., 2010; Rojas, Zapata y Grisales, 2009; Oshagbemi, 2003).

La calidad de la enseñanza está determinada por dos factores: el desempeño docente y el rendimiento en el trabajo, los cuales están influenciados por el nivel de SL del profesorado (Cantón-Mayo y Téllez-Martínez, 2017; Jordan, Miglič, Todorović, y Marič, 2017; Swaminathan y Jawahar, 2013). Si el cuerpo docente experimenta un alto nivel de satisfacción, tendrá mayor disposición para que su alumnado no solo aprenda, sino que también advierta un cambio en lo afectivo y en lo social. Las universidades con un profesorado satisfecho benefician la calidad 
de la educación y la reputación institucional (Kenney, 2010). Por el contrario, el personal docente insatisfecho, además de disminuir las probabilidades del logro educativo, influirá de manera negativa en el estado anímico de su alumnado y en la conducta de su entorno laboral (Rodríguez et al., 2010).

La literatura sobre el tema es diversa, esencialmente, en los factores que inciden sobre los niveles de SL del profesorado (Yoon, Park y Kim, 2020). En la Tabla 1 se presentan algunas propuestas de factores asociados a la SL de la plantilla docente, que han sido formuladas por diferentes autores. Por ejemplo, Aguilar-Morales, Magaña y Guzmán (2015) proponen cinco factores: condiciones de trabajo, oportunidades de promoción, reconocimiento, logro y compensación. Por su parte, Marston y Brunetti (2009) refieren cuatro factores: recompensasalario, satisfacción en el ejercicio profesional, relación con los estudiantes, colegas y administradores, y seguridad laboral-beneficios.

Tabla 1

Síntesis de investigaciones que proponen los factores de la SL del profesorado universitario

\begin{tabular}{|c|c|c|c|}
\hline N. ${ }^{\circ}$ & Autor y año & País & Factores \\
\hline 1 & $\begin{array}{l}\text { Adhikari y Barbhuiya } \\
\text { (2016) }\end{array}$ & India & $\begin{array}{l}\text { Cinco factores: pago y asignación, } \\
\text { instalaciones de bienestar, condiciones de } \\
\text { trabajo, relación con los empleados y } \\
\text { promoción. }\end{array}$ \\
\hline 2 & Park y Ramos (2018) & $\begin{array}{l}\text { Corea del } \\
\text { Sur }\end{array}$ & $\begin{array}{l}\text { Cinco factores: pago, seguridad, satisfacción } \\
\text { social, supervisión y crecimiento. }\end{array}$ \\
\hline 3 & $\begin{array}{l}\text { Aguilar-Morales et al. } \\
(2015)\end{array}$ & México & $\begin{array}{l}\text { Cinco factores: condiciones de trabajo, } \\
\text { oportunidades de promoción, reconocimiento, } \\
\text { logro y compensación. }\end{array}$ \\
\hline 4 & Marston y Brunetti (2009) & $\begin{array}{l}\text { Estados } \\
\text { Unidos }\end{array}$ & $\begin{array}{l}\text { Cuatro factores: recompensa y salario, } \\
\text { satisfacción en el ejercicio profesional, } \\
\text { relación con los estudiantes, colegas y } \\
\text { administradores, y seguridad laboral y } \\
\text { beneficios. }\end{array}$ \\
\hline 5 & $\begin{array}{l}\text { Maita, Rodríguez, } \\
\text { Gutiérrez y Núñez (2011) }\end{array}$ & Venezuela & $\begin{array}{l}\text { Siete factores: condiciones físicas y/o } \\
\text { materiales, aspecto económico, políticas } \\
\text { administrativas, relaciones sociales, } \\
\text { desarrollo del personal, desempeño de tareas } \\
\text { y relación con la autoridad. }\end{array}$ \\
\hline 6 & Tinoco et al. (2014) & Perú & $\begin{array}{l}\text { Seis factores: desarrollo profesional, relación } \\
\text { con el jefe, relación con compañeros, } \\
\text { desarrollo de la función, permanencia y } \\
\text { condiciones físicas. }\end{array}$ \\
\hline 7 & $\begin{array}{l}\text { Padilla, Jiménez } \\
\text { Ramírez (2013) }\end{array}$ & México & Un factor: la SL en general. \\
\hline 8 & $\begin{array}{l}\text { Xavier, Sugumar y Jeyaraj } \\
(2012)\end{array}$ & India & $\begin{array}{l}\text { Cinco factores: condiciones físicas de } \\
\text { trabajo, compensación, oportunidades } \\
\text { externas, política y estatus socioeconómico. }\end{array}$ \\
\hline
\end{tabular}




\begin{tabular}{|c|c|c|c|}
\hline 9 & $\begin{array}{l}\text { Tu, Bernard, Prosper y } \\
\text { Maguiraga (2005) }\end{array}$ & Inglaterra & $\begin{array}{l}\text { Seis factores: recompensa, supervisión, } \\
\text { trabajo en sí, salario, condiciones físicas del } \\
\text { trabajo y relación con colegas. }\end{array}$ \\
\hline 10 & Siddiqui y Saba (2013) & Pakistán & $\begin{array}{l}\text { Seis factores: seguridad laboral, gestión y } \\
\text { apoyo de compañeros de trabajo, salario, } \\
\text { oportunidades de promoción, condiciones del } \\
\text { trabajo y beneficios/compensación. }\end{array}$ \\
\hline 11 & Khalid et al. (2012) & Pakistán & $\begin{array}{l}\text { Cinco factores: salario, } \\
\text { supervisión, compañeros de trabajo y } \\
\text { seguridad laboral. }\end{array}$ \\
\hline 12 & Chaita (2014) & $\begin{array}{l}\text { Arabia } \\
\text { Saudita }\end{array}$ & $\begin{array}{l}\text { Cinco factores: identidad de tarea, } \\
\text { significación de la tarea, las habilidades, la } \\
\text { autonomía y la retroalimentación. }\end{array}$ \\
\hline 13 & Ara y Akbar (2016) & Pakistán & $\begin{array}{l}\text { Seis factores: tasa de remuneración, } \\
\text { promoción, objetivos personales, autonomía } \\
\text { laboral, carga de trabajo y herramientas de } \\
\text { gestión. }\end{array}$ \\
\hline
\end{tabular}

Fuente: Elaboración propia, 2020

Los trabajos de investigación muestran la configuración de la SL del profesorado universitario desde distintas perspectivas y dimensiones. Características del ambiente laboral, como la inequidad salarial, las excesivas horas de clase, la demanda de publicaciones académicas y las condiciones físicas son algunas de las causantes de la insatisfacción laboral. En efecto, los factores referidos en la Tabla 1 afectan las labores académicas y administrativas del personal docente. El proceso de medición del constructo de la SL del profesorado, sea de forma unidimensional o multidimensional, implica reflexionar sobre la naturaleza de la aplicación de los instrumentos, que deben estar específicamente diseñados y que deben cumplir con las propiedades psicométricas de validez y confiabilidad (Abad, Garrido, Olea y Ponsoda, 2006).

\section{Metodología}

\subsection{Enfoque}

Para el logro de los objetivos planteados, se empleó la metodología cuantitativa con diseño no experimental, de corte transversal y correlacional (Bernal, 2010; Hernández, Fernández y Baptista, 2014). El cumplimiento del primer objetivo implica un análisis descriptivo, mientras que para el segundo y tercero, es pertinente un análisis inferencial con el planteamiento de tres hipótesis basadas en la revisión de la literatura.

Hipótesis 1: Existe diferencia entre los niveles de SL del profesorado de la Facultad de Derecho de una universidad pública y los de otra universidad privada. 
Hipótesis 2: La formación académica, la categoría laboral y el sexo del profesorado de la Facultad de Derecho se asocian con la SL, mientras que el grupo etario no se asocia con la SL.

Hipótesis 3: Las dimensiones (reconocimiento, logro, compensación, condiciones de trabajo y oportunidades de promoción) están asociadas con el nivel global de la SL del profesorado de la Facultad de Derecho.

\subsection{Unidades de análisis}

Las unidades de análisis para el estudio estuvieron conformadas por dos grupos del personal docente ordinario de universidades peruanas: el primer grupo estuvo constituido por el profesorado que pertenece a la Facultad de Derecho y Ciencia Política de una universidad estatal o nacional (universidad pública), mientras que el segundo grupo estuvo formado por docentes que pertenecen a la Facultad de Derecho de una universidad privada de naturaleza asociativa, es decir, sin fines de lucro (universidad privada). Ambas universidades ocupan los primeros lugares de los rankings académicos, como de Scimago Institutions Rankings (SIR) y del Ranking Web de universidades (Webometrics). El criterio de inclusión estuvo determinado por la categoría de docente ordinario, así como por el ejercicio de funciones de docencia en, por lo menos, un ciclo académico en la Facultad. Previamente a la recopilación de datos, se enviaron cartas de solicitud de permiso a los directivos de las facultades de ambas universidades, con el propósito de asegurar el cumplimiento de los aspectos axiológicos establecidos por el reglamento del Comité de Ética de la Investigación. El equipo directivo favoreció la accesibilidad a la población docente, previo consentimiento informado.

En la recolección de datos se incluyó a toda la población de profesores de las facultades de Derecho de ambas universidades que cumplieran con los criterios de inclusión. La cantidad de docentes que respondieron el cuestionario quedó conformada por $n=98$ docentes (40 docentes de la universidad pública y 58 docentes de la universidad privada) que conformaron la muestra del estudio, la cual constituyó el $42.1 \%$ de la población.

\subsection{Técnicas de recolección}

El periodo de recolección de datos fue de cuatro meses (abril-julio de 2018), se utilizó la técnica de la encuesta presencial para el profesorado de la universidad pública, y en línea, vía correo electrónico, para el personal docente de la universidad privada. Se procedió de ese modo por las limitaciones de accesibilidad presencial. 
En la selección del instrumento de recolección de datos se tuvo en cuenta los resultados del estudio de revisión sobre metodologías utilizadas en los artículos de investigación cuya temática principal fue la SL del profesorado universitario (Limaymanta, 2019). El instrumento elegido fue el empleado por Aguilar-Morales et al. (2015), en función de las siguientes razones: 1) es una adaptación del cuestionario de Minnesota, compuesto por las cinco dimensiones, con ítems que se relacionan directamente con la labor de docentes universitarios; 2) los autores citados evidenciaron, además, que el instrumento cumplía con las propiedades de validez y confiabilidad; 3 ) se contó con el permiso de los autores del instrumento para su uso en este estudio.

El instrumento se adaptó al ámbito limeño siguiendo las directrices de la International Test Commission [ITC] (2017). La primera parte del instrumento presenta las variables que involucran las características sociodemográficas del profesorado universitario: edad, sexo, grado académico y categoría laboral. Luego, se encuentran los ítems de SL, cuya medición se realizó de manera indirecta a través de sus dimensiones (Abad et al., 2006). El cuestionario de SL consta de 20 ítems presentados en forma de juicios o afirmaciones, basados en la escala de Likert, con cinco alternativas de respuesta: (1) totalmente insatisfecho; (2) insatisfecho; (3) ni insatisfecho ni satisfecho; (4) satisfecho; y (5) totalmente satisfecho. A cada respuesta se le asigna un valor numérico y a la suma de las puntuaciones obtenidas en cada ítem se le denomina puntuación final o puntuación directa del participante (Abad et al., 2006; Hernández et al., 2014). Es decir, a mayor puntuación directa, mayor nivel de SL del profesorado. La descripción de cada dimensión, junto con las propiedades psicométricas del cuestionario, se muestran en la Tabla 2, mientras que la descripción del contenido de cada ítem se encuentra en la Tabla 4.

Para la validación de contenido, la escala fue sometida al dictamen de siete miembros de universidades y centros de investigación del país y del extranjero: dos especialistas del Tecnológico de Comfenalco (Colombia); uno de la Universidad Industrial de Santander (Colombia); dos profesoras de la Universidad Nacional Autónoma de México; una docente investigadora del CIPES (Portugal) y un docente de la Facultad de Ingeniería Industrial de la Universidad Nacional Mayor de San Marcos (Perú). Básicamente, cada especialista evaluó los ítems sobre el cumplimiento de tres criterios, suficiencia, claridad y coherencia, de manera dicotómica (Sí/No). Adicionalmente, se pidió que registraran alguna observación y/o sugerencia con el fin de evidenciar que el ítem midiera realmente lo que pretendía medir. 
Obtenidas las valoraciones de los expertos, se procedió a hallar el coeficiente de validez de contenido $\mathrm{V}$ de Aiken ( $\mathrm{Vj}$ ) de cada ítem (Tabla 2). La técnica asumida permite cuantificar la relevancia de cada ítem respecto al constructo que se desea medir (García-Sedeño y GarcíaTejera, 2013). Todos los ítems, a excepción del ítem 10, son significativos y mayores que 0.69 $(\alpha=0.05)$. Por consiguiente, según la tabulación brindada por Aiken (1985), el cuestionario adaptado cumple con la validación de contenido. Para el ítem 10, que no superó el mínimo valor $V$ de Aiken, se tuvo que modificar y mejorar el contenido con base en las sugerencias de los jueces. En consecuencia, el instrumento final empleado en este estudio fue el resultado de las modificaciones sugeridas por la validación de contenido.

Tabla 2

Dimensiones y propiedades psicométricas del instrumento de SL del profesorado, LimaPerú/2018

\begin{tabular}{|c|c|c|c|c|}
\hline Dimensiones & $\begin{array}{l}\text { Íte } \\
\text { m }\end{array}$ & $\mathbf{V j}$ & $\mathbf{H j}$ & $\begin{array}{c}\text { Alfa de } \\
\text { Cronbac } \\
\text { h }\end{array}$ \\
\hline \multirow{4}{*}{$\begin{array}{l}\text { (1) Reconocimiento } \\
\text { Cuando se esfuerza por hacer bien su trabajo; } \\
\text { además, hace trabajos extra, entonces necesita } \\
\text { que su superior le haga saber que lo ha notado, } \\
\text { con un comentario, una nota o una atención. }\end{array}$} & 1 & 1 & 0.800 & \multirow{4}{*}{0.906} \\
\hline & 2 & 0.810 & 0.683 & \\
\hline & 3 & 0.952 & 0.816 & \\
\hline & 4 & 1 & 0.865 & \\
\hline \multirow{4}{*}{$\begin{array}{l}\text { (2) Condiciones de trabajo } \\
\text { Características generales de los locales, } \\
\text { instalaciones, equipos, productos y demás útiles } \\
\text { existentes en el centro del trabajo. }\end{array}$} & 5 & 0.857 & 0.775 & \multirow{4}{*}{0.89} \\
\hline & 6 & 0.857 & 0.85 & \\
\hline & 7 & 0.810 & 0.677 & \\
\hline & 8 & 0.905 & 0.737 & \\
\hline \multirow{4}{*}{$\begin{array}{l}\text { (3) Logro } \\
\text { Cuando el/la docente desea realizar tareas difíciles } \\
\text { y que representen un desafío en un alto nivel. Se } \\
\text { da por la relación entre los resultados obtenidos en } \\
\text { una evaluación de desempeño y los objetivos } \\
\text { planteados. }\end{array}$} & 9 & 0.714 & 0.551 & \multirow{4}{*}{0.796} \\
\hline & 10 & 0.476 & 0.628 & \\
\hline & 11 & 0.810 & 0.693 & \\
\hline & 12 & 0.952 & 0.613 & \\
\hline \multirow{4}{*}{$\begin{array}{l}\text { (4) Compensación } \\
\text { Cuando se da un incentivo económico regular o } \\
\text { adicional como pago por la labor que se realiza. }\end{array}$} & 13 & 1 & 0.763 & \multirow{4}{*}{0.899} \\
\hline & 14 & 0.810 & 0.676 & \\
\hline & 15 & 0.857 & 0.805 & \\
\hline & 16 & 1 & 0.864 & \\
\hline \multirow{4}{*}{$\begin{array}{l}\text { (5) Oportunidades de promoción } \\
\text { Ascenso a un puesto superior incluyendo el salario } \\
\text { y responsabilidad mayor. }\end{array}$} & 17 & 0.857 & 0.839 & \multirow{4}{*}{0.919} \\
\hline & 18 & 0.714 & 0.683 & \\
\hline & 19 & 0.810 & 0.868 & \\
\hline & 20 & 0.714 & 0.900 & \\
\hline
\end{tabular}

Nota: Vj: coeficiente de validez de contenido $\mathrm{V}$ de Aiken; $\mathrm{Hj}$ : índice de homogeneidad Fuente: Elaboración propia (2019) y la definición de las dimensiones en base a Aguilar-Morales et al. (2015) 
Referente al análisis de fiabilidad, se utilizaron las medidas de consistencia interna. Dentro de los procedimientos que la Teoría Clásica de los Test ofrece, se usó el índice de homogeneidad $(\mathrm{Hj})$, basado en la correlación ítem total, y el coeficiente $\alpha$ de Cronbach. Los índices $\mathrm{Hj}$ cercanos a uno indican niveles apropiados para el test (Abad et al., 2006), mientras que el coeficiente alfa de Cronbach mayor que 0.7 indica una confiabilidad aceptable de las dimensiones (Nunnally, 1978). Con los resultados de los análisis descritos, se concluye que el instrumento de recolección cumple con las propiedades psicométricas.

\subsection{Procesamiento de análisis}

Los datos recolectados se procesaron en el programa Microsoft Excel mediante una matriz base de filas (sujetos) y columnas (variables sociodemográficas, laborales e ítems de la escala de SL). Para el tratamiento de los datos se empleó el programa IBM SPSS v. 25.0 y el Minitab v. 18.0. El análisis de resultados estuvo basado, por un lado, en los métodos de estadística descriptiva, específicamente, en medidas de resumen (promedio, desviación estándar y coeficiente de variación), tablas y gráficos de barras apiladas; por otro lado, en el análisis inferencial, mediante las pruebas de hipótesis, se usaron la prueba $t$ de Student, $U$ de Mann-Whitney, el análisis de varianza (ANOVA) y la correlación de rangos de Spearman. Para las pruebas de hipótesis, se comparó el nivel de significación $\alpha=0.05$ con el valor $p$ obtenido de los programas estadísticos usados en la determinación del rechazo o no de la hipótesis nula (Triola, 2009).

\section{Resultados}

\subsection{Análisis descriptivo}

El primer análisis responde a un resumen descriptivo de las variables sociodemográficas (Tabla 3). Se observa que, en ambas universidades, predomina el sexo masculino: 95\% en la universidad pública y $72.4 \%$ en la universidad privada. La mayoría del personal docente de la universidad pública está en la categoría de adultos mayores (57.5\%), mientras que en la universidad privada la mayoría está en la categoría de adultos (79.3\%). En cuanto a la antigüedad laboral, más del $70 \%$ de docentes de ambas universidades tiene 16 o más años de ejercicio en sus labores, lo cual evidencia una línea de carrera universitaria de amplia experiencia. Ambas universidades presentan un mayor número de personal docente ordinario en la categoría de principal, la más alta en el sistema universitario peruano, según el artículo 83 de la Ley Universitaria N. ${ }^{\circ} 30220$ (2014). 
Tabla 3

Medidas descriptivas del profesorado por universidad, Lima-Perú/2018

\begin{tabular}{lcc}
\hline \multirow{2}{*}{ Variable/categoría } & \multicolumn{2}{c}{ Universidad } \\
\cline { 2 - 3 } & Pública & Privada \\
\hline Sexo & $\%$ & $\%$ \\
Femenino & 5 & 27.6 \\
Masculino & 95 & 72.4 \\
\hline Grupo etario & $\%$ & $\%$ \\
[30-59] adulto & 42.5 & 79.3 \\
[60 a más > adulto mayor & 57.5 & 20.7 \\
\hline Antigüedad laboral (años) & $\%$ & $\%$ \\
[1 - 5] & 5 & 3.5 \\
[6 - 10] & 5 & 8.6 \\
[11 - 15] & 10 & 17.2 \\
[16 a más > & 80 & 70.7 \\
\hline Categoría laboral & $\%$ & $\%$ \\
Auxiliar & 22.1 & 30.4 \\
Asociado & 34.7 & 31.9 \\
Principal & 43.2 & 37.7 \\
\hline Título o máximo grado & $\%$ & $\%$ \\
Titulado & 30 & 13.8 \\
Magíster & 30 & 50 \\
Doctor & 40 & 36.2 \\
\hline Total & 100 & 100 \\
\hline & &
\end{tabular}

Fuente: Elaboración propia, 2020

En cuanto al análisis descriptivo de la escala de SL, la Tabla 4 muestra el análisis de la escala a nivel general, por cada dimensión y, específicamente, por cada ítem. Se aprecia que el ítem 9 (Me siento orgulloso con los logros obtenidos en mi trabajo) y el ítem 12 (Me siento a gusto por los logros que obtengo por mi trabajo) obtienen los promedios más altos, los cuales resultan los mejor valorados. En contraposición, el ítem 13 (Me siento a gusto con el sueldo que recibo por el trabajo que realizo), el ítem 15 (Me siento a gusto porque mi sueldo está por encima del promedio con otras universidades) y el ítem 16 (Siento que mi remuneración está en proporción con la cantidad de trabajo que realizo) presentan los promedios más bajos en ambos grupos. En cuanto a las cinco dimensiones, la mejor valorada por el personal docente 
de la universidad pública es el reconocimiento, con 16.3 de promedio; y para el de la universidad privada, el logro obtiene el mejor promedio, de 16.1. Para ambos grupos de docentes, la dimensión más crítica responde a la de compensación, con un promedio de 9.4 para la pública y 10.9 para la privada. A nivel general, el profesorado de ambas universidades tiene niveles similares de satisfacción, con puntuación directa promedio de 69.3 para la universidad pública y 71.8 para la privada (Tabla 4 ).

Los valores del coeficiente de variación (CV) comparan el grado de dispersión entre los distintos ítems de la escala. Para docentes de la universidad pública y de la universidad privada, los ítems 15 y 16 son los que tienen mayor heterogeneidad, con $52.5 \%$ y $48.2 \%$, respectivamente. Es decir, en estos ítems, las respuestas del profesorado fueron muy variadas, mientras que los ítems 12 y 9 son los más homogéneos, con $13.5 \%$ y $19.5 \%$, lo que indica una menor dispersión entre unas y otras.

Tabla 4

Medidas descriptivas de la SL del profesorado por universidad, Lima-Perú/2018

\begin{tabular}{|c|c|c|c|c|c|c|}
\hline \multirow[b]{2}{*}{ Ítems } & \multicolumn{3}{|c|}{ PÚBLICA } & \multicolumn{3}{|c|}{ PRIVADA } \\
\hline & $\begin{array}{c}M \\
(D E)\end{array}$ & $\begin{array}{l}C V \\
\%\end{array}$ & $\begin{array}{c}\text { Dimensión } \\
M, D E, C V \%\end{array}$ & $\stackrel{M}{(D E)}$ & $\begin{array}{l}C V \\
\%\end{array}$ & $\begin{array}{c}\text { Dimensión } \\
M, D E, C V \%\end{array}$ \\
\hline $\begin{array}{l}\text { 1. Me siento a gusto con la forma de } \\
\text { reconocimiento cuando hago un buen } \\
\text { trabajo. }\end{array}$ & $\begin{array}{c}4.02 \\
(1.17)\end{array}$ & 29.1 & \multirow{4}{*}{$\begin{array}{c}\text { Reconocimient } \\
\text { o } \\
16.3 \\
(3.79) \\
23.3\end{array}$} & $\begin{array}{c}3.71 \\
(1.08)\end{array}$ & 29.1 & \multirow{4}{*}{$\begin{array}{c}\text { Reconocimient } \\
\text { O } \\
15.2 \\
(3.67) \\
24.2\end{array}$} \\
\hline $\begin{array}{l}\text { 2. Me siento a gusto porque obtengo buena } \\
\text { reputación. }\end{array}$ & $\begin{array}{c}4.22 \\
(0.92) \\
\end{array}$ & 21.8 & & $\begin{array}{l}3.95 \\
(0.96)\end{array}$ & 24.4 & \\
\hline $\begin{array}{l}\text { 3. Me siento a gusto con el elogio que recibo } \\
\text { por realizar un buen trabajo. }\end{array}$ & $\begin{array}{c}4.05 \\
(1.11)\end{array}$ & 27.4 & & $\begin{array}{c}3.83 \\
(0.94)\end{array}$ & 24.6 & \\
\hline $\begin{array}{l}\text { 4. Me siento a gusto con el reconocimiento } \\
\text { que obtengo por mi trabajo. }\end{array}$ & $\begin{array}{c}3.95 \\
(1.22)\end{array}$ & 30.9 & & $\begin{array}{c}3.72 \\
(1.09)\end{array}$ & 29.3 & \\
\hline $\begin{array}{l}\text { 5. Me siento a gusto con las condiciones } \\
\text { materiales del lugar de mi trabajo: luz } \\
\text { artificial, equipos y ventilación. }\end{array}$ & $\begin{array}{c}3.28 \\
(1.18)\end{array}$ & 35.9 & \multirow{4}{*}{$\begin{array}{c}\text { Condiciones de } \\
\text { trabajo } \\
13.5 \\
(3.56) \\
26.4\end{array}$} & $\begin{array}{c}3.83 \\
(1.01)\end{array}$ & 26.5 & \multirow{4}{*}{$\begin{array}{c}\text { Condiciones de } \\
\text { trabajo } \\
16.1 \\
(3.33) \\
20.8\end{array}$} \\
\hline $\begin{array}{l}\text { 6. Me siento a gusto con el entorno } \\
\text { ambiental del lugar de trabajo: clima, } \\
\text { contaminación. }\end{array}$ & $\begin{array}{l}3.52 \\
(0.99)\end{array}$ & 28.0 & & $\begin{array}{l}4.03 \\
(0.95)\end{array}$ & 23.7 & \\
\hline $\begin{array}{l}\text { 7. Me siento a gusto con las condiciones } \\
\text { organizativas, como los horarios laborales y } \\
\text { descansos. }\end{array}$ & $\begin{array}{l}3.50 \\
(1.20)\end{array}$ & 34.3 & & $\begin{array}{l}3.97 \\
(0.88)\end{array}$ & 22.2 & \\
\hline $\begin{array}{l}\text { 8. Me siento a gusto con las condiciones } \\
\text { físicas, como sistema de seguridad y } \\
\text { limpieza. }\end{array}$ & $\begin{array}{l}3.22 \\
(0.97)\end{array}$ & 30.2 & & $\begin{array}{c}4.22 \\
(0.88)\end{array}$ & 20.9 & \\
\hline $\begin{array}{l}\text { 9. Me siento orgulloso con los logros } \\
\text { obtenidos en mi trabajo. }\end{array}$ & $\begin{array}{c}4.42 \\
(0.64)\end{array}$ & 14.4 & \multirow{4}{*}{$\begin{array}{c}\text { Logro } \\
16.1 \\
(2.62) \\
16.3\end{array}$} & $\begin{array}{c}4.26 \\
(0.83)\end{array}$ & 19.5 & \multirow{4}{*}{$\begin{array}{c}\text { Logro } \\
16.1 \\
(3.07) \\
19.2\end{array}$} \\
\hline $\begin{array}{l}\text { 10. Siento que me dan la oportunidad para } \\
\text { lograr mis metas profesionales. }\end{array}$ & $\begin{array}{c}3.68 \\
(1.05)\end{array}$ & 28.5 & & $\begin{array}{c}3.78 \\
(1.09)\end{array}$ & 29.0 & \\
\hline $\begin{array}{l}\text { 11. Siento que tengo las facilidades para } \\
\text { lograr las tareas asignadas. }\end{array}$ & $\begin{array}{c}3.58 \\
(1.15)\end{array}$ & 32.3 & & $\begin{array}{c}3.79 \\
(0.95)\end{array}$ & 25.1 & \\
\hline $\begin{array}{l}\text { 12. Me siento a gusto por los logros que } \\
\text { obtengo por mi trabajo. }\end{array}$ & $\begin{array}{l}4.42 \\
(0.59)\end{array}$ & 13.5 & & $\begin{array}{l}4.24 \\
(0.82)\end{array}$ & 19.5 & \\
\hline
\end{tabular}




\begin{tabular}{|c|c|c|c|c|c|c|}
\hline $\begin{array}{l}\text { 13. Me siento a gusto con el sueldo que } \\
\text { recibo por el trabajo que realizo. }\end{array}$ & $\begin{array}{c}2.25 \\
(1.13)\end{array}$ & 50.1 & \multirow{4}{*}{$\begin{array}{c}\text { Compensación } \\
9.4 \\
(3.58) \\
38.1\end{array}$} & $\begin{array}{c}2.74 \\
(1.15)\end{array}$ & 41.9 & \multirow{4}{*}{$\begin{array}{c}\text { Compensación } \\
10.9 \\
(4.37) \\
40.3\end{array}$} \\
\hline $\begin{array}{l}\text { 14. Siento que existe igualdad en el } \\
\text { reconocimiento económico frente a mis } \\
\text { colegas. }\end{array}$ & $\begin{array}{c}2.80 \\
(1.09)\end{array}$ & 39.0 & & $\begin{array}{c}3.12 \\
(1.24)\end{array}$ & 39.9 & \\
\hline $\begin{array}{l}\text { 15. Me siento a gusto porque mi sueldo está } \\
\text { por encima del promedio con otras } \\
\text { universidades. }\end{array}$ & $\begin{array}{c}2.12 \\
(1.11)\end{array}$ & 52.5 & & $\begin{array}{c}2.53 \\
(1.22)\end{array}$ & 48.2 & \\
\hline $\begin{array}{l}\text { 16. Siento que mi remuneración está en } \\
\text { proporción con la cantidad de trabajo que } \\
\text { realizo. }\end{array}$ & $\begin{array}{c}2.22 \\
(1.10)\end{array}$ & 49.4 & & $\begin{array}{c}2.47 \\
(1.19)\end{array}$ & 48.2 & \\
\hline $\begin{array}{l}\text { 17. Me siento a gusto con las oportunidades } \\
\text { para ascender. }\end{array}$ & $\begin{array}{c}3.25 \\
(1.28)\end{array}$ & 39.3 & \multirow{4}{*}{$\begin{array}{c}\text { Oportunidades } \\
\text { de promoción } \\
14.0 \\
(4.24) \\
30.3\end{array}$} & $\begin{array}{c}3.36 \\
(1.07)\end{array}$ & 31.9 & \multirow{4}{*}{$\begin{array}{c}\text { Oportunidades } \\
\text { de promoción } \\
13.7 \\
(3.94) \\
28.9\end{array}$} \\
\hline $\begin{array}{l}\text { 18. Me siento a gusto por la oportunidad de } \\
\text { dirigir a otras personas. }\end{array}$ & $\begin{array}{c}3.80 \\
(0.85) \\
\end{array}$ & 22.5 & & $\begin{array}{c}3.45 \\
(0.86) \\
\end{array}$ & 25.1 & \\
\hline $\begin{array}{l}\text { 19. Me siento a gusto porque los ascensos o } \\
\text { promociones se dan mediante criterios } \\
\text { académicos y no políticos. }\end{array}$ & $\begin{array}{c}3.52 \\
(1.26)\end{array}$ & 35.8 & & $\begin{array}{c}3.45 \\
(1.22)\end{array}$ & 35.3 & \\
\hline $\begin{array}{l}\text { 20. Siento que las oportunidades de } \\
\text { ascensos o promociones se dan de manera } \\
\text { justa y transparente. }\end{array}$ & $\begin{array}{c}3.42 \\
(1.28)\end{array}$ & 37.4 & & $\begin{array}{c}3.4 \\
(1.18)\end{array}$ & 34.9 & \\
\hline Puntuación directa de la escala de SL & $\begin{array}{c}69.3 \\
(14.5)\end{array}$ & 20.9 & & $\begin{array}{c}71.8 \\
(15.0)\end{array}$ & 20.9 & \\
\hline
\end{tabular}

Nota: $M$ : media; $D E$ : desviación estándar; $C V$ : coeficiente de variación Fuente: Elaboración propia, 2019

Según los resultados de la Figura 1, el nivel de satisfacción más frecuente en el personal docente de ambos grupos es satisfactorio (42.5\% y el $41.4 \%$ ). Sin embargo, si se analiza el grado de totalmente satisfecho, el $7.5 \%$ de docentes de la universidad pública tiene el mencionado nivel de satisfacción, que se compara con el $20.7 \%$ obtenido en la universidad privada.

Los resultados indicarían que el profesorado de la universidad privada tiene mayor nivel de SL en las dimensiones de condiciones de trabajo y compensación, en comparación con el profesorado de la universidad pública (Tabla 4); estas características están orientadas hacia las condiciones físicas, sistema de seguridad, de limpieza y el nivel salarial que perciben producto del trabajo realizado. De otro lado, sumando los resultados de insatisfechos y totalmente insatisfechos, el $10 \%$ corresponde al profesorado de la universidad pública y el $6.8 \%$ al de la privada, lo cual muestra un bajo porcentaje de insatisfacción en ambos grupos. 
Figura 1

Distribución porcentual del profesorado según nivel de SL por universidad, Lima-Perú/2018

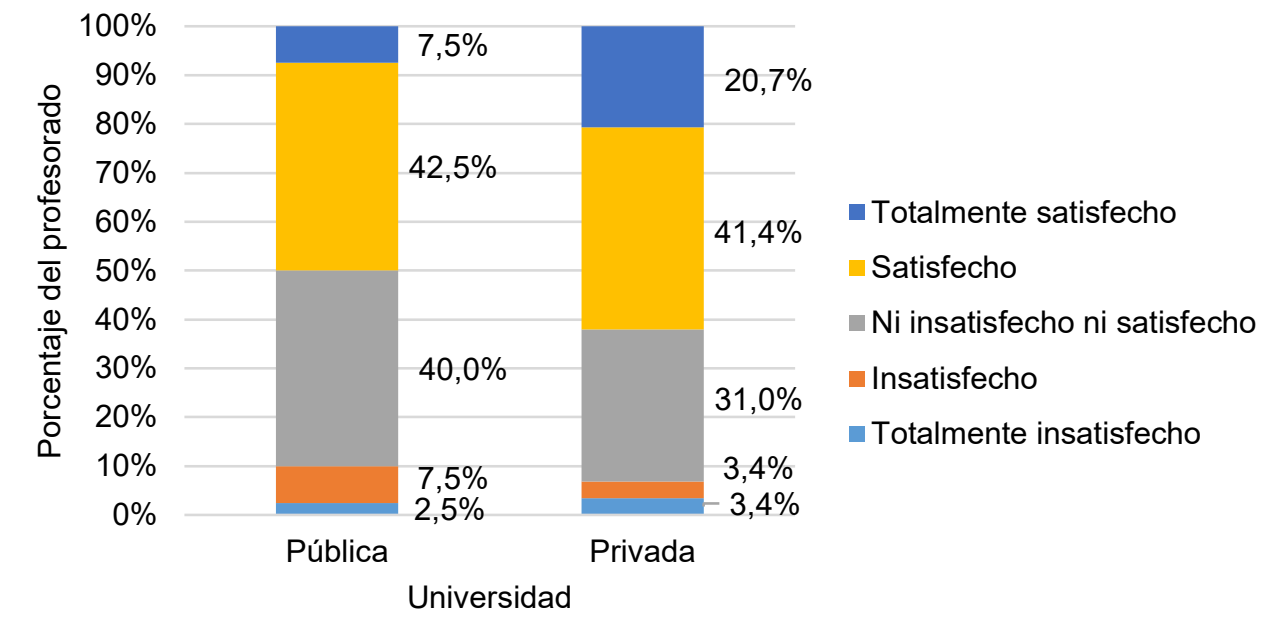

Fuente: Elaboración propia, 2019

\subsection{Análisis inferencial}

Previamente a este análisis, se realizaron las pruebas de normalidad con el fin de reconocer el tipo de método estadístico a aplicar en cada hipótesis planteada. En ese sentido, para el contraste de la Hipótesis 1, se compararon los niveles de SL entre docentes de la universidad pública y de la universidad privada. Tratándose de variables que se aproximaban a una distribución normal, se utilizó la prueba $t$ de Student para muestras independientes; caso contrario, se empleó la prueba $U$ de Mann-Whitney (UMW) (Tabla 5). El análisis de la prueba $t$ indica que no existe diferencia significativa en los niveles de SL entre el profesorado de la universidad pública $(M=69.3, D E=14.5)$ y de la privada $(M=71.8, D E=15.0), t(96)=-0.85$, $p=$.399. De ese modo, se estaría confirmando lo planteado en la Hipótesis 1. El resultado obtenido difiere de lo encontrado por Stefano, Pinto, Rossini y Limongi (2006), y Khalid et al. (2012), para quienes los académicos de universidades privadas están más satisfechos que los de las universidades públicas.

En la misma línea, se realizó la comparación de los niveles de SL entre el profesorado de ambas universidades considerando cada una de las dimensiones de la escala. En la Tabla 5 se observa que el personal docente de la universidad pública y el de la universidad privada no difieren significativamente en los niveles de la SL, en concreto, en las dimensiones de reconocimiento, logro, compensación y oportunidades de promoción. La distinción se aprecia en torno a las condiciones de trabajo, con diferencias significativas entre el profesorado de la universidad pública $(M e=14)$ y la privada $(M e=16), U=624, p<.05$. El resultado indicaría 
que el personal de enseñanza de la universidad privada presenta mejores niveles de SL que el de la universidad pública en lo que atañe a las condiciones de trabajo (condiciones materiales, luz artificial, equipos, ventilación, condiciones organizativas, horarios laborales, descansos, seguridad y limpieza).

Tabla 5

Comparación de los niveles de SL y sus dimensiones según universidad

\begin{tabular}{|c|c|c|c|c|c|}
\hline $\begin{array}{l}\text { SL } \underset{\text { dimensiones }}{y} \text { sus }\end{array}$ & Universidad & $\begin{array}{c}\text { Normalidad } \\
\text { valor } p\end{array}$ & $\begin{array}{l}\text { Tipo de } \\
\text { prueba }\end{array}$ & $\begin{array}{c}M(\text { prueba } t) \\
M e(\text { prueba UMW) }\end{array}$ & Valor $p$ \\
\hline \multirow{2}{*}{$\begin{array}{l}\text { SL-puntuación } \\
\text { directa }\end{array}$} & & .624 & $\begin{array}{c}t \text { de } \\
\text { Student }\end{array}$ & 69.3 & \multirow[b]{2}{*}{.399} \\
\hline & Privada & .200 & $\begin{array}{c}\text { /prueba } \\
F\end{array}$ & 71.8 & \\
\hline \multirow{2}{*}{ Reconocimiento } & Pública & $.004^{*}$ & $U$ de & 16.5 & \multirow{2}{*}{.107} \\
\hline & Privada & $.00002^{*}$ & $\begin{array}{l}\text { IVann- } \\
\text { Whitney }\end{array}$ & 16.0 & \\
\hline \multirow{2}{*}{$\begin{array}{l}\text { Condiciones } \\
\text { trabajo }\end{array}$} & Pública & 0.097 & Ude & 14.0 & \multirow{2}{*}{$.00001^{*}$} \\
\hline & Privada & $.00001^{*}$ & $\begin{array}{l}\text { INann- } \\
\text { Whitney }\end{array}$ & 16.0 & \\
\hline \multirow[b]{2}{*}{ Logro } & Pública & $.007^{*}$ & $U$ de & 16.0 & \multirow{2}{*}{.546} \\
\hline & Privada & $.00001^{*}$ & $\begin{array}{c}\text { Mann- } \\
\text { Whitney }\end{array}$ & 16.0 & \\
\hline \multirow[b]{2}{*}{ Compensación } & Pública & .084 & $\begin{array}{c}t \text { de } \\
\text { Student }\end{array}$ & 9.4 & \multirow[b]{2}{*}{.083} \\
\hline & Privada & .200 & $\begin{array}{c}\text { /prueba } \\
F \\
\end{array}$ & 10.9 & \\
\hline \multirow{2}{*}{$\begin{array}{l}\text { Oportunidades de } \\
\text { promoción }\end{array}$} & Pública & .079 & $\begin{array}{c}U \text { de } \\
\text { Mann- }\end{array}$ & 14.5 & \multirow{2}{*}{.611} \\
\hline & Privada & $.049^{*}$ & Whitney & 14.0 & \\
\hline
\end{tabular}

Nota: ${ }^{*} p<.05, M=$ media, $M e=$ mediana

Fuente: Elaboración propia, 2019

Para reconocer la asociatividad de los factores sociodemográficos y laborales, se realizó la comparación de las puntuaciones directas. Se consideró la formación académica, categoría laboral, grupo etario y sexo. Se aplicó el ANOVA y se verificó si la formación académica y la categoría laboral afectan los niveles de SL. Previamente, se comprobó el cumplimiento de los supuestos de normalidad de errores y homogeneidad de varianzas. En la Tabla $6 \mathrm{~A}$ se observa que la formación académica, $F(2,95)=3.09, p=.305$ y la categoría laboral de los participantes, $F(2,95)=3.09, p=.433$, no afectan los niveles de SL del profesorado. Los resultados indican que la más alta formación académica o la más alta categoría laboral en el sistema universitario peruano no se asocian, necesariamente, a mayores niveles de SL. Contrariamente, Oshagbemi (2003) y Eyupoglu y Saner (2009) encontraron que el rango académico afecta los 
niveles de satisfacción asociados con el avance, la compensación, los compañeros de trabajo y la variedad para los académicos.

Tabla 6

\begin{tabular}{|c|c|c|c|c|c|c|}
\hline \multicolumn{7}{|c|}{ A. Comparación de los niveles de SL según formación académica y categoría laboral } \\
\hline \multicolumn{2}{|c|}{ Verificación de supuestos } & \multicolumn{5}{|c|}{ Variable respuesta: Puntuación directa de la SL } \\
\hline \multirow{3}{*}{$\begin{array}{l}\text { Normalidad } \\
\text { valor } p\end{array}$} & \multirow{3}{*}{$\begin{array}{l}\text { Homogeneidad } \\
\text { valor } p\end{array}$} & Factor & $n$ & $M$ & $\boldsymbol{F}$ & Valor $p$ \\
\hline & & \multicolumn{2}{|c|}{ Formación académica } & & & \multirow{4}{*}{.305} \\
\hline & & Titulado & 20 & 75.2 & \multirow{3}{*}{1.203} & \\
\hline \multirow[t]{2}{*}{0.310} & \multirow[t]{2}{*}{0.624} & Magíster & 41 & 70.3 & & \\
\hline & & Doctor & 37 & 69.0 & & \\
\hline \multicolumn{7}{|c|}{ Categoría laboral } \\
\hline \multirow{3}{*}{0.200} & \multirow{3}{*}{0.728} & Auxiliar & 26 & 70.7 & \multirow{3}{*}{0.844} & \multirow{3}{*}{.433} \\
\hline & & Asociado & 37 & 68.7 & & \\
\hline & & Principal & 35 & 73.2 & & \\
\hline \multicolumn{7}{|c|}{ B. Comparación de los niveles de SL según grupo etario y sexo } \\
\hline $\begin{array}{l}\text { Normalidad } \\
\quad \text { valor } p\end{array}$ & Factor & Grupo & $n$ & $\begin{array}{c}M \text { (prueb } \\
t \text { ) } \\
\text { Me } \\
\text { (prueba } \\
U M M \text { ) }\end{array}$ & $\begin{array}{l}\text { Tipo de } \\
\text { prueba }\end{array}$ & Valor $p$ \\
\hline 0.137 & \multirow[b]{2}{*}{ Grupo etario } & Adulto & 63 & 69.6 & \multirow{2}{*}{$\begin{array}{c}t \text { de } \\
\text { Student/ } \\
\text { prueba } F\end{array}$} & \multirow[b]{2}{*}{.285} \\
\hline 0.334 & & $\begin{array}{l}\text { Adulto } \\
\text { mayor }\end{array}$ & 35 & 72.9 & & \\
\hline $0.010^{*}$ & \multirow{2}{*}{ Sexo } & Masculino & 80 & 74.0 & $\begin{array}{l}U \text { de } \\
\text { Mann- }\end{array}$ & \multirow{2}{*}{.089} \\
\hline $0.00001^{*}$ & & Femenino & 18 & 65.0 & Whitney & \\
\hline
\end{tabular}

Nota: ${ }^{*} p<.05 ; n=$ tamaño de muestra; $F=$ estadístico de prueba $F$

Fuente: Elaboración propia, 2020

La Tabla 6B presenta los resultados de la prueba $t$ y de la prueba $U$ de Mann-Whitney. Con un $5 \%$ de nivel de significación, se concluye que no existe una diferencia significativa de los niveles de $S L$ entre el profesorado adulto $(M=69.6, D E=15.3)$ y adulto mayor $(M=72.9$, $D E=13.7), t(96)=-1.08, p=.285$. En consecuencia, el grupo etario al que pertenece el cuerpo docente no se asocia con la SL, resultado que coincide con los estudios de Tu et al. (2005) y Oshagbemi (2003). Sin embargo, en el caso de Toker (2011), sus resultados muestran que en grupos de edad, a partir de los 61 años, los niveles promedio de SL son significativamente más altos que entre los 21-40 años. De otro lado, no existe diferencia en los niveles de SL entre el profesorado de sexo masculino $(M e=74)$ y el profesorado femenino $(M e=65), U=$ $535 ; p=.089$. Es decir, el sexo no se asocia con los niveles de SL del profesorado, al igual que lo evidenciado por El Badawy y Magdy (2015), Toker (2011) y Oshagbemi (2003). Todos estos resultados no confirman la Hipótesis 2, la cual estaba basada en suposiciones previas. 
Adicionalmente, se pidió a los participantes que valoraran, a nivel global, su nivel de SL con una escala estadística de intervalo del 1 al 10, donde la mayor puntuación significa un mayor nivel de SL. Estos datos se recolectaron a fin de evidenciar si alguna de las dimensiones se relaciona con el nivel global de la SL.

La Tabla 7 muestra los resultados del análisis de correlación de rangos de Spearman. Se prueba que existe una correlación directa y significativa de la SL, a nivel global, con las cinco dimensiones propuestas en el cuestionario. El valor de la correlación más alta se da con las oportunidades de promoción $r_{s}(98)=.752, p<.05$. La relación positiva de la SL con cada dimensión indica que a mayor nivel de puntuación de cada dimensión, mayor será el nivel de la SL a nivel global del profesorado universitario. Lo anterior confirma la Hipótesis 3.

Dichos resultados coinciden con los de Vega y Moyano (2010), quienes evidenciaron una correlación directa y significativa entre la SL con cada una de sus dimensiones. De todas las dimensiones expuestas, Maita et al. (2011), Eyupoglu y Saner (2009) y Xavier et al. (2012) resaltaron que la satisfacción con la compensación serviría para retener al personal docente de buen desempeño y garantizar la mejora de la calidad de su enseñanza. Contrariamente, la insatisfacción con esta dimensión produciría consecuencias negativas en la productividad profesional del personal de enseñanza. Esto evidencia que se debe poner una atención estratégica a esta dimensión por parte de las instancias correspondientes.

Tabla 7

Correlación entre la SL a nivel global y las cinco dimensiones

\begin{tabular}{ccccccc}
\hline \multicolumn{2}{c}{ Dimensiones de la SL } & Reconocimiento & $\begin{array}{c}\text { Condiciones } \\
\text { de trabajo }\end{array}$ & Logro & Compensación & $\begin{array}{c}\text { Oportunidades } \\
\text { de promoción }\end{array}$ \\
\hline $\begin{array}{c}\text { SL a nivel } \\
\text { global }\end{array}$ & $\boldsymbol{r}_{\boldsymbol{s}}$ & $.670^{*}$ & $.544^{*}$ & $.727^{*}$ & $.621^{*}$ & $.752^{*}$ \\
\cline { 2 - 6 } & $\begin{array}{c}\text { valor } \\
\boldsymbol{p}\end{array}$ & & & & & \\
& & & $.00001 \rightarrow$ & &
\end{tabular}

Nota: ${ }^{*} p<.05 ; r_{s}$ : coeficiente de correlación de rangos de Spearman

Fuente: Elaboración propia, 2020

\section{Conclusiones}

La pregunta de investigación sobre cuáles son los factores que configuran la SL en el profesorado de una universidad pública y de una universidad privada, ubicadas en Lima, fue respondida a través del logro de los tres objetivos específicos. El primer objetivo remite a que el $42.5 \%$ del profesorado de la universidad pública se encuentra satisfecho laboralmente, mientras que dicho porcentaje para docentes de la universidad privada es $41.4 \%$. Se comprobó 
que los ítems peor valorados por el profesorado de ambas facultades son los que pertenecen a la dimensión compensación, que manifiesta insatisfacción con los ingresos percibidos por el ejercicio de la docencia. Es decir, no existe igualdad en el reconocimiento económico entre colegas, sino insatisfacción, dado que las remuneraciones están por debajo del promedio de otras universidades y que no estarían en proporción a la cantidad de trabajo que realizan.

En el segundo objetivo específico se concluye que no existe una diferencia significativa entre los niveles de SL del profesorado de las facultades de Derecho de ambas universidades. No obstante, si se compara la SL con cada una de las cinco dimensiones (reconocimiento, condiciones de trabajo, logro, compensación y oportunidades de promoción) se encuentra diferencia significativa de los niveles de SL en la dimensión condiciones de trabajo. Es decir, el profesorado de la Facultad de Derecho de la universidad privada tiene mayor satisfacción por las condiciones de trabajo que ofrece su centro de labor en comparación con el grupo de docentes de la universidad pública. Tales valoraciones responderían a las condiciones materiales como luz artificial, equipos y ventilación; condiciones del entorno ambiental; condiciones organizativas de horarios laborales; condiciones de seguridad y limpieza.

Respecto al último objetivo, se concluye que la formación académica, la categoría laboral, el grupo etario y el sexo no se asocian con los niveles de SL. Estos resultados evidencian que no necesariamente el profesorado con mayor grado académico o con mayor categoría laboral se sentirá más satisfecho laboralmente. Ni que el hecho de ser hombre o mujer o tener mayor edad significará mayores niveles de SL. Asimismo, se demostró que las cinco dimensiones presentan una relación positiva y alta, estadísticamente significativa, con los niveles de SL del profesorado.

Considerando los resultados obtenidos, se presentan algunas propuestas para incrementar la SL del profesorado a partir del análisis de sus dimensiones. Concerniente al reconocimiento, resulta indispensable que las autoridades de cada facultad o unidad académica valoren la trayectoria de la carrera docente, la experiencia, las competencias y los conocimientos alcanzados por los profesores. Avanzar en esa perspectiva presupone transparencia y valoración de la meritocracia mediante incentivos a las mejores prácticas profesionales, la innovación, la investigación y la excelencia profesional. Para reforzar la dimensión de compensación, el Estado peruano y las universidades privadas podrían propiciar una atención estratégica, vía incrementos salariales progresivos, dada su gravitación en la SL del profesorado universitario, a fin de garantizar la mejora de la calidad educativa. 
Sobre la dimensión oportunidades de promoción, el artículo 83 de la Ley Universitaria N. ${ }^{\circ} 30220$ (2014) establece los requisitos para la progresión de la carrera docente. En ese sentido, para que el profesorado tenga altos niveles de SL se debe garantizar que las promociones se den de manera justa y transparente, y con criterios académicos y no políticoinstitucionales. Referente a la dimensión condiciones de trabajo, se sugiere que el personal a cargo de la enseñanza universitaria cuente con el asesoramiento de las instituciones donde laboran para que conozcan sus deberes, derechos y condiciones laborales. Asimismo, el estado y las universidades deberán intervenir en la gestión de mejora de la infraestructura, promover la comunicación interna entre docentes y generar empatía para incrementar los niveles de SL y, por tanto, de la calidad de la enseñanza.

Esta investigación presentó limitaciones, sobre todo en la recolección de datos, por la resistencia de cierto sector del profesorado de la universidad pública a responder al cuestionario de SL, así como por la imposibilidad de aplicar la encuesta presencial al profesorado docentes de la universidad privada, por lo que se realizó mediante el correo electrónico. Este estudio se concentró expresamente en el profesorado de una unidad académica por cada universidad, por lo que abre las puertas a la posibilidad de futuras investigaciones que aborden otras titulaciones, o que involucren a la totalidad del profesorado de una o varias universidades. Finalmente, se pudo constatar que existen numerosas investigaciones sobre la SL del personal docente y diferentes propuestas de las dimensiones que la conforman, pero pocas intentan comparar entre dos o más poblaciones distintas. Sería muy útil si las investigaciones venideras establecieran semejanzas y diferencias entre el profesorado de distintas procedencias o sectores económicos, relacionando la SL con otras variables como el absentismo laboral, estrés laboral, síndrome de burnout, apoyo directivo, resiliencia, salud mental, entre otros.

\section{Agradecimientos}

En primer lugar, los autores agradecen al profesorado de ambas universidades, quienes, voluntaria y desinteresadamente, respondieron el cuestionario de SL. En segundo lugar, dan las gracias a los siete jueces que participaron en la fase de validación de contenido del cuestionario de SL. Por último, agradecen a la Facultad de Letras y Ciencias Humanas de la Universidad Nacional Mayor de San Marcos por promover la investigación científica, y a todas las personas que directa o indirectamente estuvieron involucradas en esta investigación, lo cual permitió culminar con éxito este artículo. 


\section{Referencias}

Abad, Francisco, Garrido, Jesús, Olea, Julio y Ponsoda, Vicente. (2006). Introducción a la psicometría. Madrid, España: Universidad Autónoma de Madrid.

Adhikari, Kingshuk y Barbhuiya, Juber Hussain. (2016). Mapping job satisfaction of central university teachers. Splint International Journal of Professionals, 3(11), 22-28. Recuperado de https://search.proquest.com/openview/d0da3b41718b088a40a67d8b5d5aa2fa/1?pqorigsite $=$ gscholar $\& \mathrm{cbl}=2044944$

Aguilar-Morales, Norma, Magaña, Deneb y Guzmán, Candelaria. (2015). Satisfacción laboral en profesores investigadores universitarios. Revista Internacional Administración \& Finanzas, 8(6), 11-28. Recuperado de https://ssrn.com/abstract=2661784

Aiken, Lewis. (1985). Three coefficients for analyzing the reliability and validity of ratings. Educational and Psychological Measurement, 45(1), 131-142. doi: https://doi.org/10.1177/0013164485451012

Álvarez-Flores, David. (2007). Satisfacción y fuentes de presión laboral en docentes universitarios de Lima metropolitana. Persona, 10, 49-97. doi: https://doi.org/10.26439/persona2007.n010.913

Ara, Kaukab y Akbar, Aisha. (2016). A study of impact of moonlighting practices on job satisfaction of the university teachers. Bulletin of Education and Research, 38(1), 101116. Recuperado de http://pu.edu.pk/images/journal/ier/PDFFILES/8 Kaukab\%20\&\%20Aisha v38 1 2016.pdf

Bernal, César. (2010). Metodología de la investigación, administración economía, humanidades y ciencias sociales ( $3^{\mathrm{a}}$ ed). Bogotá, Colombia: Pearson Educación. Recuperado de http://abacoenred.com/wp-content/uploads/2019/02/El-proyecto-deinvestigaci\%C3\%B3n-F.G.-Arias-2012-pdf.pdf

Cantón-Mayo, Isabel y Téllez-Martínez, Sonia. (2016). La satisfacción laboral y profesional de los profesores. Revista Lasallista de Investigación 13(1), 214-226. doi: https://doi.org/10.22507/rli.v13n1a18

Cantón-Mayo, Isabel y Téllez-Martínez, Sonia. (2017). La satisfacción en el desempeño profesional de los docentes de educación infantil y educación primaria. Un estudio de caso. Profesorado: Revista de currículum y formación del profesorado, 21(1), 279-292. Recuperado de https://recyt.fecyt.es/index.php/profesorado/article/view/62505

Chaita, Mercy. (2014). The impact of employee attitudes on job satisfaction: A comparative study of university professors in the northwest region of England. International Journal on Global Business Management \& Research, 3(1), 2-12. Recuperado de http://www.rajalakshmi.org/ijgbmr/downloads/IJGBMRAug14.pdf 
Cranny, Charles, Smith, Patricia Cain y Stone, Eugene. (1992). Job satisfaction: how people feel about their jobs and how it affects their performance. New York, United States of America: Lexington Books. Recuperado de https://www.worldcat.org/title/jobsatisfaction-how-people-feel-about-their-jobs-and-how-it-affects-theirperformance/oclc/25008324

Duche-Pérez, Aleixandre Brian, Gutiérrez-Aguilar, Olger Albino y Paredes-Quispe, Fanny Miyahira. (2019). Satisfacción laboral y compromiso institucional en docentes universitarios peruanos. Revista Conrado, 15(70), 15-24. Recuperado de https://conrado.ucf.edu.cu/index.php/conrado/article/view/1099

El Badawy, Tarek y Magdy, Mariam. (2015). Assessing the impact of emotional intelligence on job satisfaction: An empirical study on faculty members with respect to gender and age. International Business Research, 8(3), 67-78. doi: https://doi.org/10.5539/ibr.v8n3p67

Eyupoglu, Serife Zihni y Saner, Tulen. (2009). Job satisfaction: Does rank make a difference?. African Journal of Business Management, 3(10), 609-615. Recuperado de https://academicjournals.org/journal/AJBM/article-abstract/25AB6FF18399

Frías, Rosario. (2006). Estudio de satisfacción del profesorado en la Universidad Pública Española. Empiria. Revista de Metodología de Ciencias Sociales, (11), 175-201. doi: https://doi.org/10.5944/empiria.11.2006.1113

García-Sedeño, Manuel y García-Tejera, María Cristina. (2013). Estimación de la Validez de Contenido en una Escala de Valoración del Grado de Violencia de Género Soportado en Adolescentes. Acción Psicológica, 10(2), 41-58. doi: http://dx.doi.org/10.5944/ap.10.2.11823

Guëll Malet, Luisa. (2014). Estudio de la satisfacción laboral de los maestros (Tesis doctoral). Universitat Internacional de Catalunya, España. Recuperado de https://www.tdx.cat/bitstream/handle/10803/293783/Tesi Luisa G\%C3\%BCell Malet.p df? sequence $=1$

Hernández, Roberto, Fernández, Carlos y Baptista, Pilar. (2014). Metodología de la investigación (6 $6^{\mathrm{a}}$ ed.). Distrito Federal, México: Mc. Graw Hill.

International Test Commission [ITC]. (2017). The ITC Guidelines for Translating and Adapting Tests ( $\quad 2^{\text {nd }} \quad$ ed). Recuperado de https://www.intestcom.org/files/guideline test adaptation 2ed.pdf

Jordan, Gasper, Miglič, Gozdana, Todorović, Ivan y Marič, Miha. (2017). Psychological Empowerment, Job Satisfaction and Organizational Commitment Among Lecturers in Higher Education: Comparison of Six CEE Countries. Organizacija, 50(1), 17-32. doi: https://doi.org/10.1515/orga-2017-0004

Kenney, Mathew. (2010). Professor pay dissatisfaction: A comparison of academic entrepreneurs and traditional faculty. Journal of Business Studies Quarterly, 1(3), 24-30. Recuperado de https://ssrn.com/abstract=1627902 
Khalid, Salman, Zohaib, Muhammad y Mahmood, Babak. (2012). Job Satisfaction among Academic Staff: A Comparative Analysis between Public and Private Sector Universities of Punjab, Pakistan. International Journal of Business and Management, 7(1), 126-136. doi: $\underline{\text { https://doi.org/10.5539/ijbm.v7n1p126 }}$

Ley Universitaria N³0220. (2014). El Peruano, N 12914, 9 de julio. Editora Perú. Recuperado de https://diariooficial.elperuano.pe/pdf/0021/ley-universitaria-30220.pdf

Limaymanta, Cesar Halley. (2019). Tendencias de diseños metodológicos en las publicaciones indexadas sobre la satisfacción laboral del profesorado universitario. Revista Electrónica Educare, 23(3), 1-23. doi: https://doi.org/10.15359/ree.23-3.6

Locke, Edwin. (1969). What is job satisfaction? Organizational Behavior and Human Performance, 4(4), 309-336. doi: https://doi.org/10.1016/0030-5073(69)90013-0

Maita, Haydeé, Rodríguez, Daysi, Gutiérrez, Lucas y Núñez, Linda. (2011). Factores de satisfacción laboral en los docentes del núcleo Bolívar de la Universidad de Oriente. SABER. Revista Multidisciplinaria del Consejo de Investigación de la Universidad de Oriente, 23(2) 147-151. Recuperado de https://www.redalyc.org/pdf/4277/427739446008.pdf

Mapesela, Mabokang y Hay, Driekie. (2006). The effect of change and transformation on academic staff and job satisfaction: A case of a South African University. Higher Education, 52(4), 711-747. doi: https://doi.org/10.1007/s10734-004-6821-7

Marston, Susan y Brunetti, Gerald. (2009). Job Satisfaction of Experienced Professors at a Liberal Arts College. Education, 130(2), 323-347. Recuperado de http://web.wilkes.edu/jennifer.edmonds/MBA 513/job\%20satisfaction.pdf

Morán, Gabriela y Alvarado, Darío. (2010). Métodos de investigación. México: Pearson Educación.

Recuperado

de https://mitrabajodegrado.files.wordpress.com/2014/11/moran-y-alvarado-metodos-deinvestigacion-1ra.pdf

Nunnally, Jum. (1978). Psychometric Theory (2 ${ }^{\text {nd }}$ ed.). New York: McGraw-Hill.

Oshagbemi, Titus. (2003). Personal correlates of job satisfaction: Empirical evidence from UK universities. International Journal of Social Economics, 30(12), 1210-1232. doi: https://doi.org/10.1108/03068290310500634

Padilla, Laura Elena, Jiménez, Leonardo y Ramírez, María de los Dolores. (2013). La satisfacción laboral en el personal académico y su relación con la intención de abandonar la profesión. Perfiles Educativos, 35(141), 8-25. doi: https://doi.org/10.1016/S0185$\underline{2698(13) 71832-4}$

Park, Chung y Ramos, Hazel. (2018). The Level of Job Satisfaction as Indicator of Work ValueFulfillment Trend in Higher Education Systems. International Journal of Advanced Science and Technology, 112(2018), 43-56. doi: https://doi.org/10.14257/ijast.2018.112.05 
Rodríguez, Daysi, Núñez, Linda y Cáceres, Alfonso. (2010). Estudio comparativo de la satisfacción laboral universitaria en el núcleo de Anzoátegui de la Universidad de Oriente. Investigación y Postgrado, 25(1) 63-80. Recuperado de https://dialnet.unirioja.es/servlet/articulo?codigo $=5330851$

Rojas, Maylén, Zapata, Johana y Grisales, Hugo. (2009). Síndrome de burnout y satisfacción laboral en docentes de una institución de educación superior. Medellín, 2008. Revista Facultad Nacional de Salud Pública, 27(2), 198-210. Recuperado de https://revistas.udea.edu.co/index.php/fnsp/article/view/924/1871

Siddiqui, Anam y Saba, Iram. (2013). Determining the job satisfaction level of the academic staff at tertiary academic institutes of Pakistan. International Journal of Information, Business and Management, 5(3), 42-53. Recuperado de https://ijibm.elitehall.com/index4.htm

Ssesanga, Karim y Garrett, Roger. (2005). Job satisfaction of university academics: Perspectives from Uganda. Higher Education, 50(1), 33-56. doi: https://doi.org/10.1007/s10734-004-6346-0

Stefano, Silvio, Pinto, María Cristina, Rossini, Viviane y Limongi, Ana Cristina. (2006). Satisfação da qualidade de vida no trabalho com relação aos fatores biopsicossociais e organizacionais: Um estudo comparativo entre docentes das universidades pública e privada. Revista Ibero Americana de Estratégia, 5(2), 35-44. Recuperado de https://www.redalyc.org/pdf/3312/331227108004.pdf

Swaminathan, Samanvitha y Jawahar, David. (2013). Job Satisfaction as a Predictor of Organizational Citizenship Behavior: An Empirical Study. Global Journal of Business Research, 7(1), 71-80. Recuperado de https://ssrn.com/abstract=2147886

Tinoco, Oscar, Quispe, Carlos y Beltrán, Victor. (2014). Cultura organizacional y satisfacción laboral en la Facultad de Ingeniería Industrial en el marco de la acreditación universitaria. Industrial Data, 17(2), 56-66. doi: https://doi.org/10.15381/idata.v17i2.12048

Toker, Boran. (2011). Job satisfaction of academic staff: An empirical study on turkey. Quality Assurance in Education, 19(2), 156-169. doi: https://doi.org/10.1108/09684881111125050

Triola, Mario. (2009). Estadística $\left(10^{a}\right.$ ed.). México: Pearson Educación. Recuperado de https://www.uv.mx/rmipe/files/2015/09/Estadistica.pdf

Tu, Leafy, Plaisent, Michel, Bernard, Prosper, y Maguiraga, Lassana. (2005). Comparative age differences of job satisfaction on faculty at higher education level: China and Taiwan. International Journal of Educational Management, 19(3), 259-267. doi: https://doi.org/10.1108/09513540510591039

Vega, Andrés y Moyano, Emilio. (2010). Adicción al trabajo, satisfacción laboral y familiar en académicos de una universidad estatal chilena. Salud \& Sociedad, 1(3), 222-232. doi: https://doi.org/10.22199/S07187475.2010.0003.00006 
Xavier, Joseph, Sugumar, Dharmalingam y Jeyaraj, P. (2012). A study on job satisfaction among the academicians across the self-financing arts and science colleges in Tiruchirappalli, South Tamil Nadu, India. International Journal of Management Research and Review, 2(10), 1764-1776. Recuperado de http://ijmrr.com/admin/upload data/journal angapan 10oct12.pdf

Yoon, Jiyoung, Park, Soojung y Kim, Dae-Jin. (2020). Assessing the effects of higher-education factors on the job satisfaction of engineering graduates in Korea. Sustainability, 12(8). doi: https://doi.org/10.3390/SU12083342 
Revista indizada en
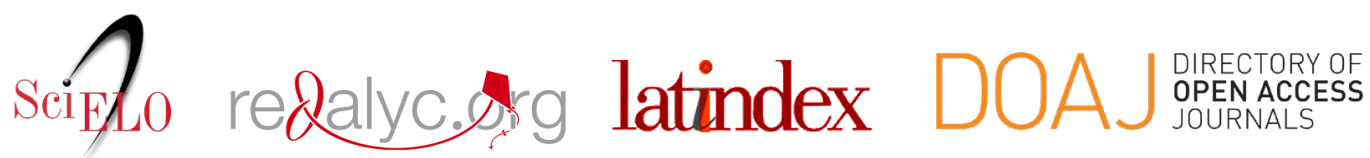

Distribuida en las bases de datos:

- Dialnet

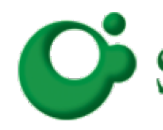
SHERPA/RoMEO

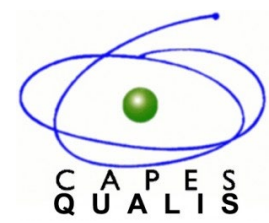

MIAR 